\title{
Numerical Analysis of Acoustic Propagation inside Multiple Liquids
}

\author{
Shervin Foroughi, Vahid Karamzadeh, Mohsen Habibi, Muthukumaran Packirisamy \\ Optical Bio-Microsystem Laboratory, Mechanical and Industrial Engineering Department, Concordia University \\ Sir George Williams Campus 1455 De Maisonneuve Blvd. W., Montreal, Canada \\ s_foroug@encs.concordia.ca; vahid.karamzadeh@mail.concordia.ca; mohs_hab@encs.concordia.ca; \\ mpackir@encs.concordia.ca
}

\begin{abstract}
This paper describes numerical analysis of acoustic propagation inside multiple Newtonian liquids. The applied acoustic pressure is generated by high intensity focused ultrasound transducer. Analysis is performed by implementation of linear wave equation as well as heat transfer equations for fluids. Simulation is done in COMSOL Multiphysics as a finite element software. Investigation is focused on study of sound effects, temperature and pressure gradient, in focal region inside three distinct liquids with various properties. Results clearly illustrate the dependency of heat transfer and pressure distribution in focal area on physical properties of medium it passes through as well as wave frequency.
\end{abstract}

Keywords: Acoustic, Heat Transfer, Temperature rise, Transducer, HIFU, Intensity.

\section{Introduction}

High intensity ultrasound energy has been frequently employed as an operating energy in various devices for different applications. This energy can be used for plastic welding in automotive, electronic appliance, and medical equipment industries [1]. It also is being utilized in acoustic compressors, refrigerators, and other industrial processes [2].

In therapeutic methods for non-invasive ablation of tumours, high intensity focused ultrasound (HIFU) is used [3]. Possibility of performing treatment operation from outside of body on a tissue is the main advantages of this method over other thermal ablation techniques [4].

This paper describes numerical analysis of acoustic propagation inside multiple Newtonian liquids in presence of changes in physical properties of liquid and solid elements which wave is passing through as well as wave frequency. It is believed that this physic brings a better understanding of the possibility and flexibility of industrial application of high intensity focused ultrasound.

The applied acoustic pressure is generated by HIFU transducer. This aperture concentrates pressure wave at a certain point inside fluid which is called focal point and the following studies are performed in this region. As a matter of fact, analysis is done by implementation of basic governing equations for sound propagation and heat transfer. The result of acoustic study is heat energy due to absorbance of acoustic by bulk of liquid during transmission of ultrasound. Therefore, heat transfer study can be performed in time domain in presence of the computed heat source. Next, investigation of results are proceeded by implementing characteristics of multiple liquids in the simulation, changing the material of a container as well as well as altering acoustic source input parameters. In subsequent sections theory and simulation and consequential results are discussed.

\section{Theory and Numerical Analysis}

\subsection{Acoustic Theory}

Pressure acoustics, frequency domain interface of COMSOL Multiphysics 5.2 is used to model the stationary acoustic field in a liquid. In this simulation the linear homogeneous Helmholtz equation in absence of shear waves and nonlinear acoustic wave propagation effects is solved [5]. It is assumed that the geometry of model elements are symmetrical and liquid medium has uniform acoustic properties. Therefore, 2D axisymmetric assumption for acoustic field model will be an acceptable approximation that leads to reducing computation time. As a result, the equation in cylindrical coordinate is presented as [6]: 


$$
\frac{1}{\rho \omega^{2}}\left[\nabla^{2}+\left(\frac{\omega}{c}\right)^{2}\right] \mathbf{p}(r, z)=0
$$

Where $\omega$ is angular velocity, $\mathbf{p}$ is an acoustic pressure. Parameters $\rho$ and $c$ are density and speed of sound related to liquid. Here $r$ and $z$ are the radial and axial coordinates of axisymmetric cylindrical coordinate in which $\nabla^{2}$ is defined as:

$$
\nabla^{2}=\frac{1}{r} \frac{\partial}{\partial r} r \frac{\partial}{\partial r}+\frac{\partial^{2}}{\partial z^{2}}
$$

The major effect of acoustic propagation inside liquid is the thermal energy produced due to absorption of ultrasound plane-wave by liquid that yield to rising medium temperature. The temperature distribution depends on a convection and conduction properties of liquid. The amount of generated ultrasound power per unit volume $\boldsymbol{Q}_{A}$ is obtained by applying following expression [7]:

$$
\boldsymbol{Q}_{A}=2 \alpha_{A b} I=\frac{2 \alpha_{A b}}{\omega^{2} \rho c}\left\langle\left(\frac{\partial \mathbf{p}}{\partial t}\right)^{2}\right\rangle
$$

Where, $\alpha_{A b}$ is the local absorption coefficient of the liquid, $I$ is the local acoustic intensity, and the brackets defines time average over one acoustic cycle.

\subsection{Heat Transfer Theory}

To model heat transfer in a liquid medium, following equation is used [8]:

$$
\rho C_{p} \frac{D \boldsymbol{T}}{D t}=-(\nabla \cdot q)-(\tau: \nabla V)-\left(\frac{\partial \ln \rho}{\partial \ln T}\right)_{p} \frac{D p}{D t}+\boldsymbol{Q}_{A}
$$

Here, $\rho$ is the density of liquid, $C_{p}$ is the specific heat capacity at constant pressure, $\boldsymbol{T}$ is the absolute temperature, $q$ is the heat flux from conduction, $p$ is the pressure, $V$ is the velocity vector as well as $\tau$ which is the viscous stress tensor and $\tau: \nabla V$ refers to irreversible rate of internal energy increase per unit volume by viscous dissipation in Newtonian fluid, and $\boldsymbol{Q}_{A}$ is additional heat source due to acoustic pressure.

By considering the liquid as an incompressible fluid, the term $\left(\frac{\partial \ln \rho}{\partial \ln T}\right)_{p} \frac{D p}{D t}$ can be eliminated [8]. Therefore, the final form of "4" for incompressible fluid will be:

$$
\rho C_{p} \frac{D \boldsymbol{T}}{D t}=-(\nabla \cdot q)-(\tau: \nabla V)+\boldsymbol{Q}_{A}
$$

This equation is used to study fluids heat transfer in a time domain by COMSOL.

\subsection{Numerical Analysis and Model}

For determination of the acoustic effects, pressure and temperature, in liquid media a finite difference analysis is performed in a two-step process:

1- Solving acoustic equation "1" in frequency domain to find acoustic pressure as well as heat energy due to absorption of sound wave by liquid.

2- Obtaining temperature rise by solving heat transfer equation "5" incorporated with computed heat source, in time domain. Multiple time steps are considered in order to assure the accuracy of computation. The final results are presented for 0.2 second time step in total process duration of 120 seconds.

To obtain accurate results at focal region, pressure and temperature gradient, the fine mesh with size $\lambda / 6$ ( $\lambda$ is the wavelength) and coarser mesh with size $\lambda / 4$ are chosen for focal region and other parts of model respectively [5]. 
The 3D model of a system and its components are shown in "Fig. 1". The model includes two parts: Acoustic apparatus and Fluid container. Fluid container is divided into two compartments, one contains liquid of experiments and other one is filled by pure water which provides medium for operation of transducer.

The sound source specification has been selected based on Model H-106, manufactured by Sonic Concepts Inc. This device is a spherically focused piezoceramic transducer with a focal length of $63.2 \mathrm{~mm}$ and an aperture of $64.0 \mathrm{~mm}$ which is driven at a frequency of $2.0 \mathrm{MHz}$. the focal region is in oval shape with length of $5.72 \mathrm{~mm}$ and girth of $0.76 \mathrm{~mm}$. This type of HIFU transducer is used in biomedical applications and need to be immersed in water during operation for transmitting the wave to target medium.

The space divider of container which will be called Divider from now on is going to be selected from different materials which according to the approach is being followed in present work, will be changed in multiple simulations respectively.
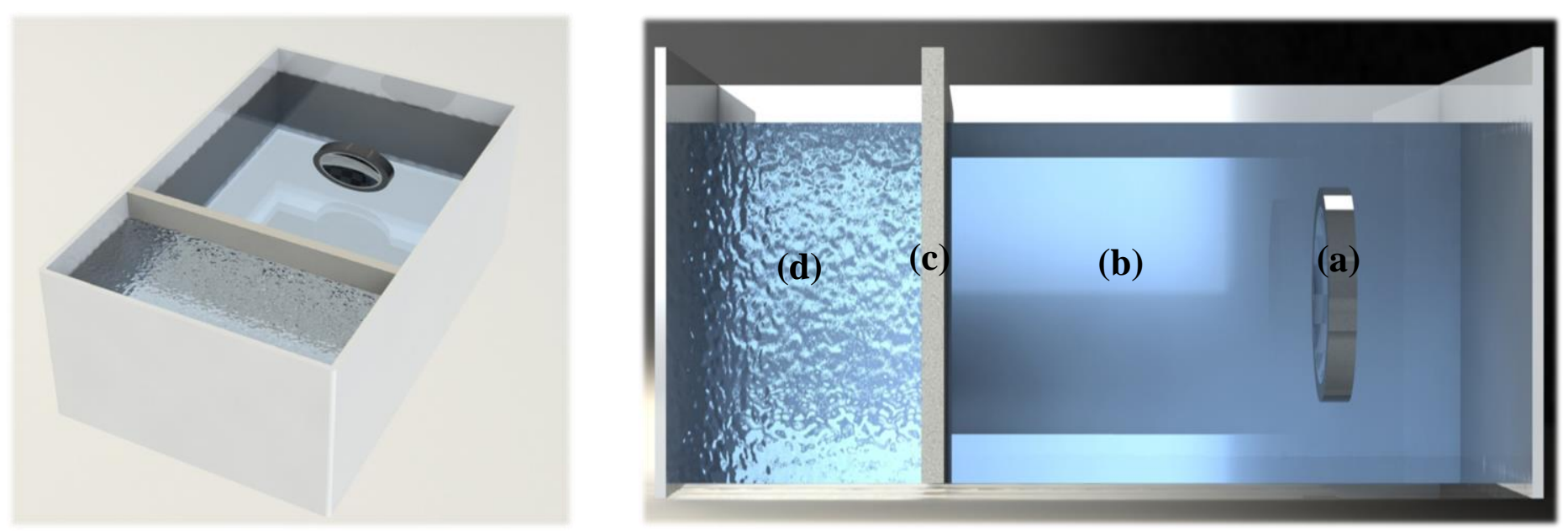

Fig. 1: 3D Model of Acoustic System.

(a): HIFU Transducer, (b): Water medium that transducer moves inside, (c): Divider, (d): Liquid of experiment.

\subsection{Materials}

"Table 1" introduces three incompressible liquids as well as their properties that will be considered as a target medium of study. All fluids have been assumed to be Newtonian and their listed properties remain constant against temperature gradient during a time.

Table 1: Liquids' Properties [9-14].

\begin{tabular}{|c|c|c|c|c|c|c|}
\hline Liquid & $\begin{array}{c}\text { Density } \\
\left(\mathrm{Kg} / \mathrm{m}^{3}\right)\end{array}$ & $\begin{array}{c}\text { Dynamic } \\
\text { Viscosity } \\
(\mathrm{Cp})\end{array}$ & $\begin{array}{c}\text { Specific Heat } \\
\left(\mathrm{J} / \mathrm{Kg} .{ }^{\circ} \mathrm{C}\right)\end{array}$ & $\begin{array}{c}\text { Heat } \\
\text { Conductivity } \\
\left(\mathrm{W} / \mathrm{m} .{ }^{\circ} \mathrm{C}\right)\end{array}$ & $\begin{array}{c}\text { Sound } \\
\text { Speed } \\
(\mathrm{m} / \mathrm{s})\end{array}$ & $\begin{array}{c}\text { Attenuation } \\
(\mathrm{Np} / \mathrm{m}) \\
@ 2 \mathrm{MHz}\end{array}$ \\
\hline Fresh Water @ $25^{\circ} \mathrm{C}$ & 997 & 0.895 & 4180 & 0.6076 & 1485 & 0.1012 \\
\hline $\begin{array}{c}\text { Sea Water @25 } 5^{\circ} \mathrm{C} \\
(36 \% \text { salinity) }\end{array}$ & 1023 & 0.968 & 3955 & 0.6414 & 1534 & 0.1420 \\
\hline Glycerin @ 25 ${ }^{\circ} \mathrm{C}$ & 1260 & 1490 & 2608 & 0.2853 & 1909 & 10 \\
\hline
\end{tabular}

In addition to study of acoustic propagation in multiple liquids, the effect of altering divider's material will be investigated. "Table 2" shows list of materials that are used as a divider. 
Table 2: Divider Materials [15].

\begin{tabular}{|c|c|c|c|c|c|}
\hline Material & $\begin{array}{c}\text { Density } \\
\left(\mathrm{Kg} / \mathrm{m}^{3}\right)\end{array}$ & $\begin{array}{c}\text { Sound } \\
\text { Speed } \\
(\mathrm{m} / \mathrm{s})\end{array}$ & $\begin{array}{c}\text { Acoustic } \\
\text { Impedance } \\
\left(\mathrm{Kg} / \mathrm{m}^{2} . \mathrm{s}\right)\end{array}$ & $\begin{array}{c}\text { Attenuation } \\
(\mathrm{Np} / \mathrm{m}) \\
@ 2 \mathrm{MHz}\end{array}$ & $\begin{array}{c}\text { Thickness } \\
(\mathrm{mm})\end{array}$ \\
\hline ABS, grey & 1070 & 2170 & $2.31 \times 10^{6}$ & 20.82 & $1 / 2{ }^{\prime}$ \\
\hline Acrylic, Clear & 1190 & 2750 & $3.26 \times 10^{6}$ & 11.79 & $1 / 2{ }^{\prime}$ \\
\hline Polycarbonate, Clear & 1180 & 2270 & $2.69 \times 10^{6}$ & 45.87 & $1 / 2{ }^{\prime}$ \\
\hline
\end{tabular}

\section{Results}

"Fig. 3" illustrates the amplitude of acoustic pressure as a function of radial distance along a horizontal axis extended from focal points in liquids: water, seawater and Glycerin, in presence of ABS divider. Presenting different acoustic impedance for liquids causes to distinct magnitudes of acoustic pressure at focal points.

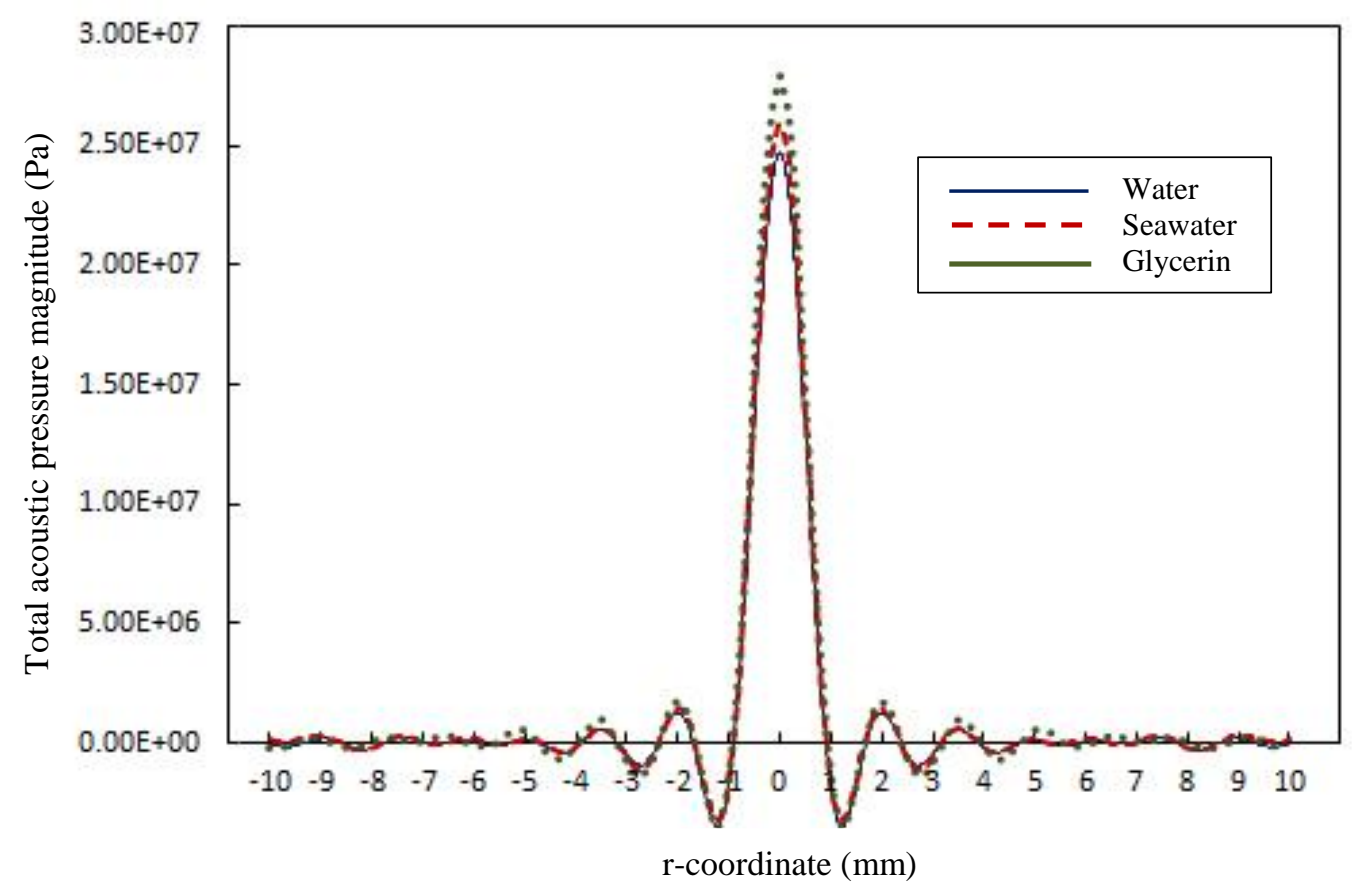

Fig. 3: Total acoustic pressure profile along the radial axis extended from focal points inside different liquids.

The acoustic intensity profile at focal region in multiple liquids again in presence of ABS divider is represented in "Fig. 4". The highest magnitude of intensity occurs at focal point. Diversity in intensities amplitudes are due to different acoustic impedance of fluids. Peak points on graphs happened at focal point and they reveal its location. The demonstrated variations in focal places are caused by sound speed alteration while transmission through the divider and fluids. This matter is completely consistent with Snell's law for acoustic wave travelling in various mediums. 


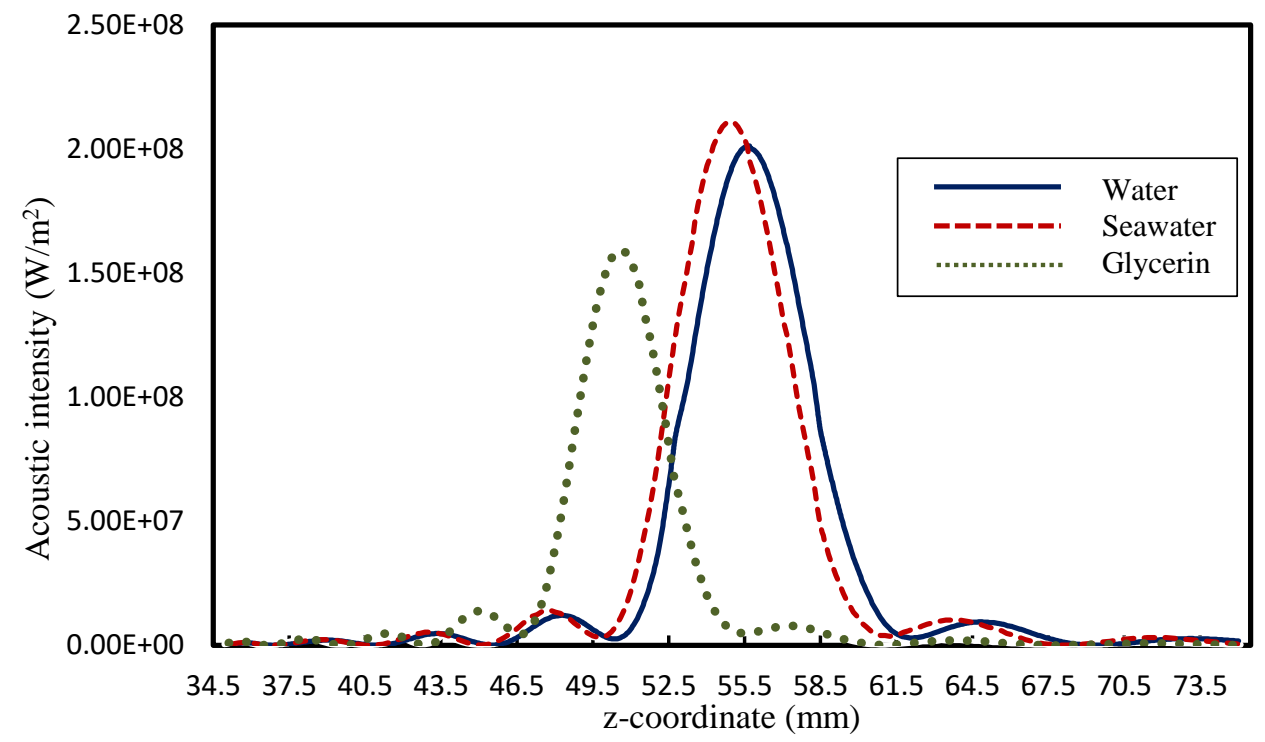

Fig. 4: Acoustic intensity profile in multiple liquids.

"Fig. 5" presents the acoustic intensity field with respect to frequency. Fluid medium and divider material are considered water and ABS respectively. Although, results demonstrates the growth of intensity by frequency increment, the focal region size declines while frequency rise. Furthermore, this figure shows that the most of acoustic energy is concentrated at focal point. 


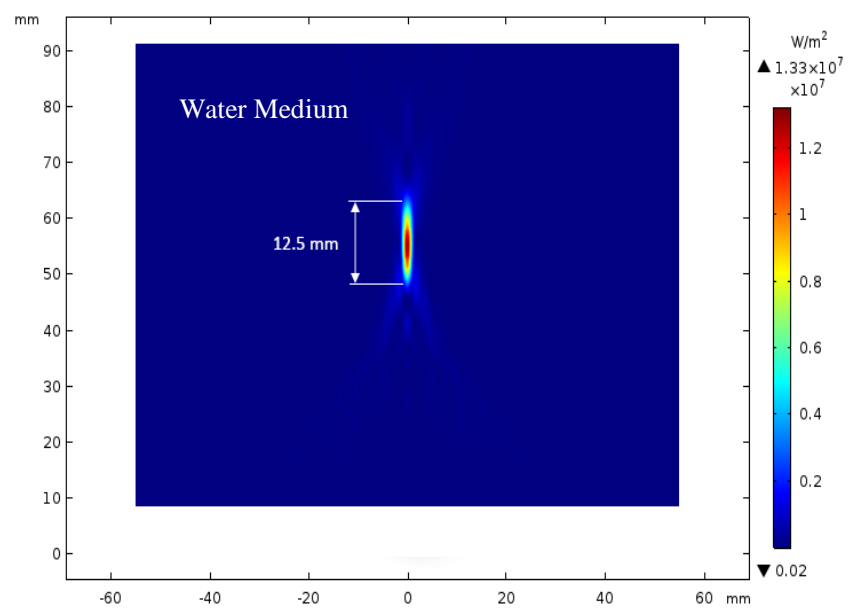

Frequency: 1.0 MHZ, Focal Intensity: $13.3 \mathrm{MW} / \mathrm{m}^{2}$,

Focal Region Length: $12.5 \mathrm{~mm}$

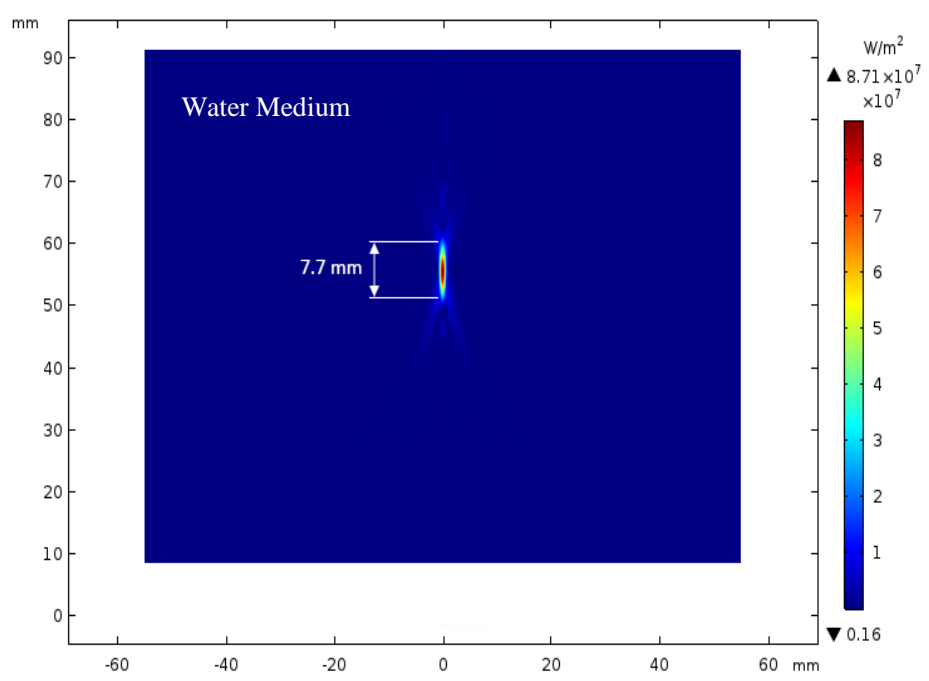

Frequency: 1.6 MHZ, Focal Intensity: $87.1 \mathrm{MW} / \mathrm{m}^{2}$, Focal Region Length: $7.7 \mathrm{~mm}$

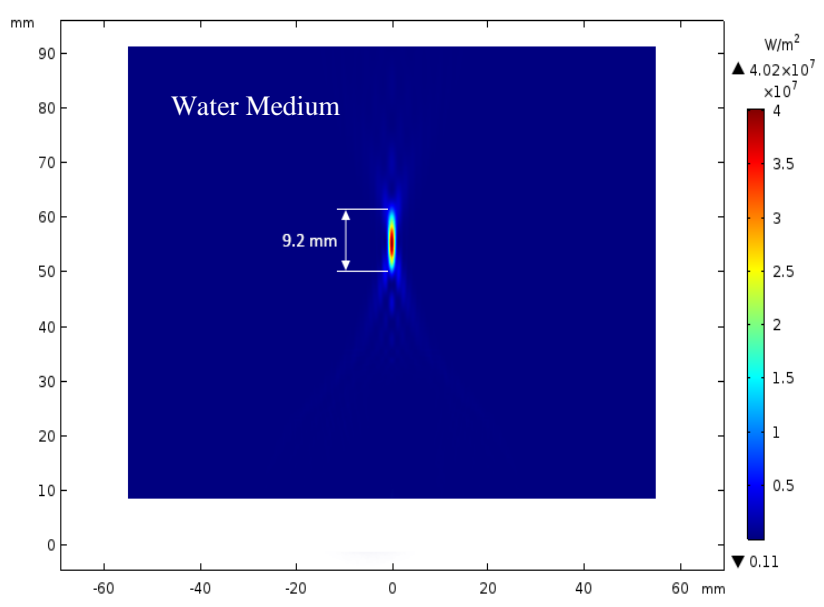

Frequency: 1.3 MHZ, Focal Intensity: $40.2 \mathrm{MW} / \mathrm{m}^{2}$, Focal Region Length: $9.2 \mathrm{~mm}$

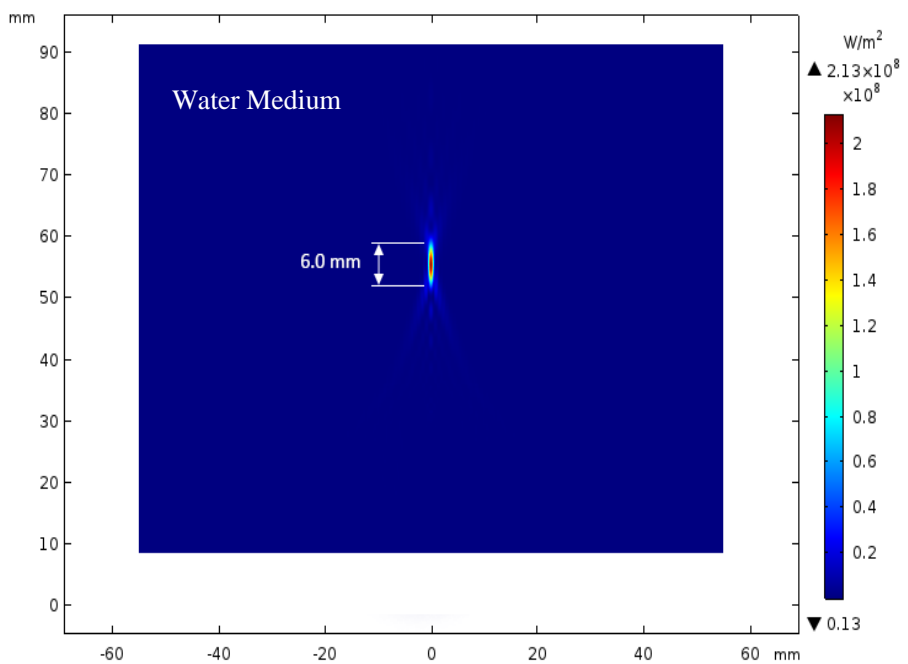

Frequency: 2.0 MHZ, Focal Intensity: $213.2 \mathrm{MW} / \mathrm{m}^{2}$, Focal Region Length: $6.0 \mathrm{~mm}$

Fig. 5: Acoustic intensity field and focal region size for different frequencies.

The effect of using multiple dividers on heat transfer at focal point is shown in "Fig. 6". Heat is induced by absorption of ultrasound wave in the water. The acoustic wave is exerted at frequency of $2 \mathrm{MHz}$ for 2 seconds. Assuming a constant liquid medium for all three case studies, temperature rise differences are due to variation of multiple dividers' acoustic impedance property. 


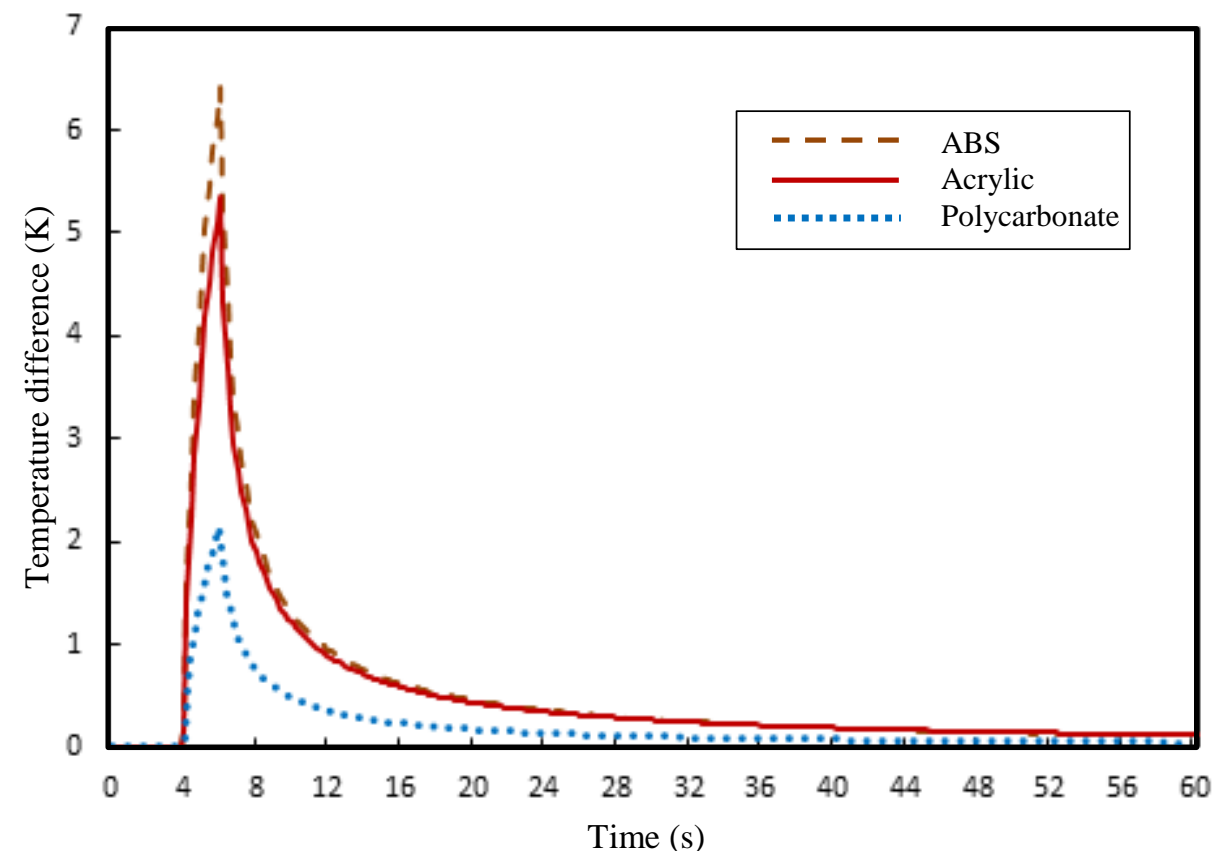

Fig. 6: Heat transfer and temperature rise at focal point inside water by applying $2 \mathrm{MHz}$ acoustic wave for 2 seconds.

By replacement of liquids in presence of a permanent ABS divider, the attenuation property will have the major role on temperature magnitude inside fluid. "Fig. 7" displays the variation of temperature at focal point inside three mediums: (a) Water and Seawater, (b) Glycerin. Results show for the similar acoustic condition, high increment in temperature happens in Glycerin because of having attenuation factor about 100 times greater than the one for Water and Seawater.

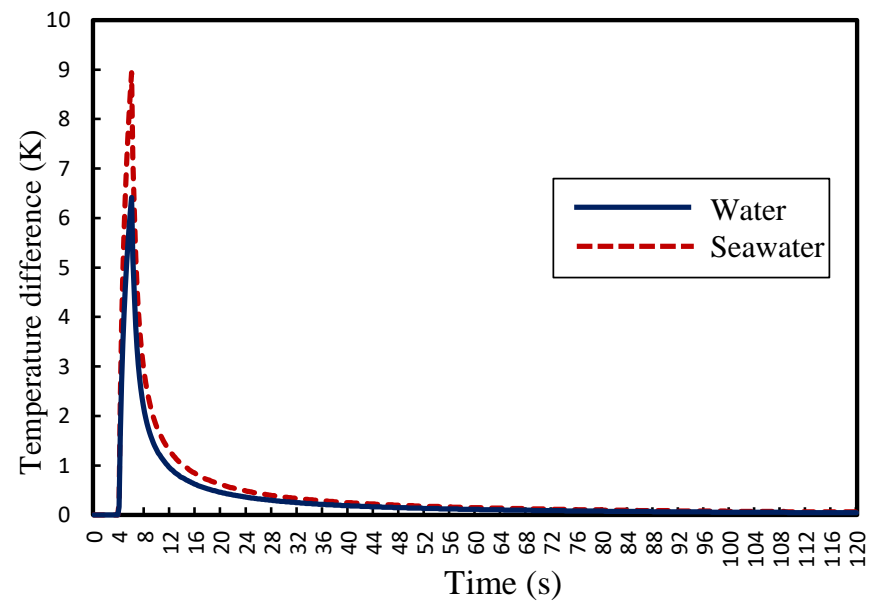

(a)

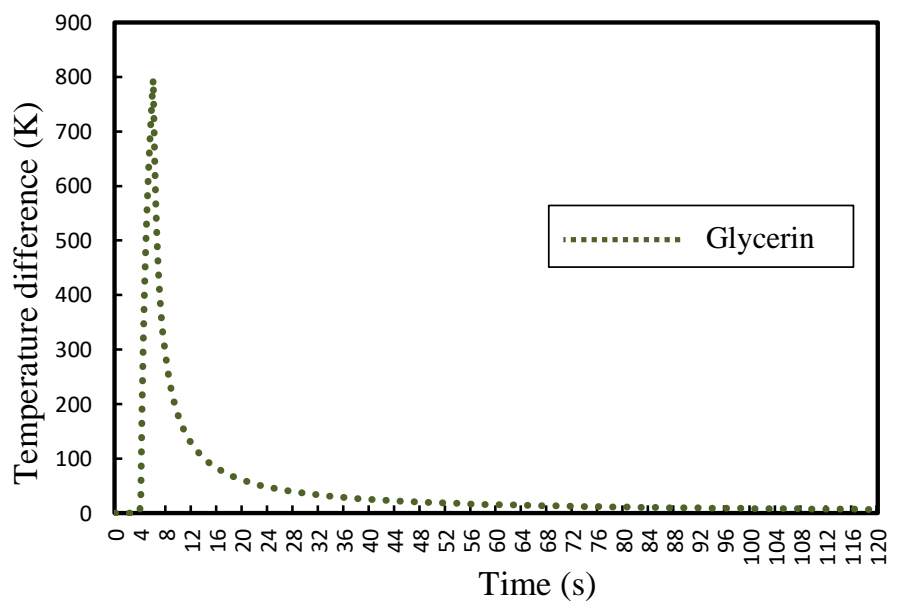

(b)

Fig. 7: Heat transfer and temperature rise at focal point inside a) Water and Seawater b) Glycerin in presence of ABS divider by applying $2 \mathrm{MHz}$ acoustic wave for 2 seconds.

\section{Conclusion}

Transmission effects of high intensity focused ultrasound (HIFU) in multiple liquids have been studied. In order to cut down the computation time and computer expenses, 2D axisymmetric simulation has been accomplished. Results showed wave frequency and fluid properties have major influence on acoustic and thermal effects in liquid medium. Wave frequency manipulation affects the focal region size and amplitude of Intensity. Present investigation illustrated acoustic pressure field varied by liquid's acoustic impedance. On the other hand, during travelling acoustic wave among different materials the wave power decreased as a result of acoustic impedance alteration. Furthermore, magnitude of temperature 
rise in liquid directly depends on absorption coefficient of fluid so that a small variation in attenuation causes the high order change in heat energy. It should be mentioned the accuracy of computed results depend on alteration of fluids characterisation during the process, simulation time step and model's mesh size. In this study properties of liquids have been considered constant during the process. By decreasing the time step, changes occurred in temperature at focal point is estimated less than $2.9 \%$. By modifying the focal region mesh size to $\lambda / 8$ and $\lambda / 10$ the changes in pressure and temperature at this region are estimated less than $3.6 \%$ and $3.8 \%$ respectively.

\section{References}

[1] H. Nguye, H. Nguyen, J. Uan and D. Wang, "A NONRATIONAL B-spline profiled horn with high displacement amplification for ultrasonic welding," Journal of Ultrasonics, vol. 83, no. 4, pp. 2063-2071, 2014.

[2] W. Han, J. Liu, D. Chong and J. Yan, "Analysis of Temperature Field in a High-Intensity Ultrasonic Chamber," Journal of Thermophysics and Heat Transfer, vol. 30, no. 4, pp. 745-753, 2016.

[3] M. Solovchuk, T.W.H. Sheu and M. Thiriet, "Simulation of nonlinear Westervelt equation for the investigation of acoustic streaming and nonlinear propagation effects," Acoustical Society of America, vol. 134, no. 5, pp. 39313942, 2013.

[4] G. T. Haar and C. Coussios, "High intensity focused ultrasound: physical principles and devices," International Journal of Hyperthermia, vol. 23, no. 2, pp.89-104, 2007.

[5] COMSOL Multiphysics 5.2a, application ID: 12659, Focused Ultrasound Induced Heating in Tissue Phantom. [Online]. Available: https://www.comsol.com/model/focused-ultrasound-induced-heating-in-tissue-phantom-12659

[6] F. B. Jensen, W. A. Kuperman, M. B. Porter, and H. Schmidt, Computational ocean acoustics, 2nd ed. Springer: New York, 2011, pp. 65-154.

[7] Huang, Holt, Cleveland, and Roy, "Experimental validation of a tractable numerical model for focused ultrasound heating in flow-through tissue phantoms," The Journal of the Acoustical Society of America, vol. 116, no. 4, pp. 2451-2458, 2004.

[8] R. B. Bird, W. E. Stewart, and E. N. Lightfoot, Transport Phenomena, 2nd ed. John Wiley \& Sons: New York, 2007, pp. 333-361.

[9] J. V. Sengers, J. T. R. Watson, "Improved International Formulations for the Viscosity and Thermal Conductivity of Water Substance," Journal of Physical and Chemical Reference Data, vol. 15, no. 4, pp. 1291-1314, 1986.

[10] 26th ITTC Specialist Committee on Uncertainty Analysis, ITTC recommended procedures, "Fresh Water and Seawater Procedure," International Towing Tank Conference, 7.5-02-01-03, Rev.02, 2011.

[11] W.A. Kuperman, R. Philippe, "Underwater Acoustics," Springer Handbook of Acoustics, Springer-Verlag: New York, 2007.

[12] J. J. Markham, R. T. Beyer, R. B. Lindsay, "Absorption of Sounds in Fluids," Reviews of Modern Physics, vol. 23, no. $4,1951$.

[13] J. P. Abulencia, L. Theodore, "Fluid Flow for the Practicing Chemical Engineer," New York: John Wiley \& Sons Inc., 2009, pp. 555-556.

[14] J. M. M. Pinkerton, "A pulse method for the Measurement of Ultrasonic Absorption in Liquids-Results for Water," Nature Publishing Group, vol. 160, no. 4056, pp. 128-129, 1947.

[15] A. R. Selfridge, "Approximate Material Properties in Isotropic Materials," IEEE Transactions on Sonics and Ultrasonics, vol. su-32, no. 3, pp. 381-394, 1985. 